\title{
Fractional flow reserve-guided percutaneous coronary intervention: where to after FAME 2?
}

\author{
This article was published in the following Dove Press journal: \\ Vascular Health and Risk Management \\ 3 December 2015 \\ Number of times this article has been viewed
}

Tim P van de Hoef'

Martijn Meuwissen ${ }^{2}$

Jan J Piek'

'AMC Heartcentre, Academic Medical Center, University of Amsterdam, Amsterdam, ${ }^{2}$ Amphia Hospital, Breda, the Netherlands
Correspondence: Tim P van de Hoef AMC Heartcentre, Academic Medical Center, University of Amsterdam, Room B2-213,

Meibergdreef 9, II 05 AZ, Amsterdam, the Netherlands

Tel +3I 205667883

Fax +3I 206962609

Email t.p.vandehoef@amc.uva.nl

\begin{abstract}
Fractional flow reserve (FFR) is a well-validated clinical coronary physiological parameter derived from the measurement of coronary pressures and has drastically changed revascularization decision-making in clinical practice. Nonetheless, it is important to realize that FFR is a coronary pressure-derived estimate of coronary blood flow impairment. It is thereby not the same as direct measures of coronary flow impairment that determine the occurrence of signs and symptoms of myocardial ischemia. This consideration is important, since the FAME 2 study documented a limited discriminatory power of FFR to identify stenoses that require revascularization to prevent adverse events. The physiological difference between FFR and direct measures of coronary flow impairment may well explain the findings in FAME 2. This review aims to address the physiological background of FFR, its ambiguities, and its consequences for the application of FFR in clinical practice, as well as to reinterpret the diagnostic and prognostic characteristics of FFR in the light of the recent FAME 2 trial outcomes.
\end{abstract}

Keywords: fractional flow reserve, coronary flow, stable ischemic heart disease

\section{Introduction}

The introduction of fractional flow reserve (FFR) into the armamentarium of the interventional cardiologist in the 1990s has retrospectively been one of the few practice-changing innovations in decision-making regarding coronary revascularization in stable ischemic heart disease patients. ${ }^{1}$ This physiological index derived from the measurement of coronary pressure has since gathered substantial clinical outcomes data through randomized clinical trials, supporting its beneficial characteristics when used for revascularization decision-making compared with clinical decision-making based on visual interpretation of the coronary angiogram alone. ${ }^{2-4}$ Notwithstanding the documented benefit of FFR-guided intervention over angiographic guidance, and its importance to simplify clinical decision-making in the catheterization laboratory, the clinical data supporting FFR have led interventionalists to apply FFR as a dichotomous gold-standard test for myocardial ischemia and to blindly adhere to FFR for revascularization decision-making in a red light/green light fashion. In contrast with this clinical application of FFR, the recent Fractional Flow Reserve Guided Percutaneous Coronary Intervention Plus Optimal Medical Therapy Versus Optimal Medical Therapy (FAME 2) trial documented that the majority of stenoses deemed hemodynamically significant by FFR do not suffer from adverse events when revascularization is deferred, whereas stenoses deemed nonsignificant by FFR are still prone to adverse cardiac events. As such, FAME 2 has shed new light on the diagnostic and prognostic characteristics of this red light/green light approach to FFR-guided revascularization in contemporary 
clinical practice and raises questions regarding contemporary revascularization guidelines. ${ }^{5,6}$ This review aims to address the physiological background of FFR, its ambiguities, and its consequences for the application of FFR in clinical practice, as well as to reinterpret the diagnostic and prognostic characteristics of FFR in the light of the recent FAME 2 trial outcomes.

\section{The physiology behind FFR}

Young et al were the first to propose the ratio between flow in a stenosed coronary artery and flow in the same coronary artery without the stenosis as an index to quantify the impairment of coronary flow induced by the stenosis. ${ }^{7}$ Although conceptually valid, the application of such an index requires the assessment of flow in the same coronary artery both with and without the stenosis, and thus by definition requires revascularization. Pijls et al later used a simplified model of the relationship between coronary pressure and flow during maximal vasodilation to extend this flow-based concept to the pressure-derived concept of FFR. ${ }^{1}$ FFR assumes the ratio of distal coronary pressure - measured by means of a pressure sensor-equipped guide wire - to aortic pressure - measured by means of the guiding catheter - at maximal coronary vasodilation to reflect the ratio of maximal flow in a stenosed artery to maximal flow in the same artery without the stenosis. Hence, FFR was initially introduced as a pressure-derived proxy measure of relative coronary flow reserve (CFR). ${ }^{8}$ As such, it is important to consider that FFR is not the same as direct measures of coronary flow from which it was derived, ${ }^{9}$ and the use of a ratio of coronary pressures as a surrogate of the actual ratio of coronary flows is based on several physiological assumptions that are important to consider when routinely applying this tool to the clinical setting. ${ }^{10-12}$

\section{Stable and minimal microvascular resistance: the holy grail in FFR assessment}

As described in the experimental validation of FFR, a ratio of coronary pressures can only serve as an estimate of the ratio of flow in a stenosed artery to flow in the same artery without the stenosis in the presence of stable and minimal coronary resistance. ${ }^{1}$ During resting conditions, coronary flow is autoregulated and is independent of coronary perfusion pressure in the physiological perfusion pressure range, by a process termed coronary autoregulation. ${ }^{11,12}$ Further, in resting conditions, coronary flow is adapted to myocardial demand by metabolic vasodilation. Coronary autoregulation and metabolic adaptation are interrelated processes that together maintain stable coronary flow during the resting state (Figure 1). Hence, in normal resting conditions, no direct relationship between coronary pressure and flow is present, and resting cycle-averaged pressure measurements therefore cannot be used as a direct estimate of the impairment of coronary flow. For coronary pressure to be used for estimation of flow impairment, coronary autoregulation should be completely abolished. ${ }^{1,13}$ At such a state of maximal coronary vasodilation, the relationship between coronary pressure and flow is incremental linear - it is straight in the physiological range of perfusion pressure and has a nonzero pressure intercept - and thereby allows estimation of flow impairment from the measurement of coronary pressures. ${ }^{11,14}$

This condition of maximal vasodilation, frequently referred to as maximal hyperemia, is aimed to be achieved by the administration of potent coronary vasodilators, most commonly adenosine. ${ }^{15}$ However, coronary autoregulation is a complex and multifactorial process regulated by several mediators. ${ }^{16}$ Although adenosine is an important mediator in this process, it is far from able to abolish all vasoconstrictor tone. ${ }^{16-18}$ While, in the concept of FFR, the administration of adenosine is assumed to lead to a stable and minimal magnitude of coronary resistance, equivalent in magnitude across all patients, in reality, adenosine only abolishes part of the coronary vasomotor tone, the magnitude of which depends on the balance between all coronary vasoconstrictors in the individual patient. In this regard, it is important to realize that medication (such as beta-blockers, alpha-blockers, and ACE inhibitors), comorbid conditions (among which are obesity, chronic obstructive pulmonary disease, and renal failure), and factors like smoking and caffeine use may also drastically interfere with the achievement of maximal hyperemia, and therefore lead to important variability in the extent of "maximal" hyperemia achievable in the individual patient. The variability of this maximal hyperemic state is illustrated by the numerous studies that have aimed to document a single dose and administration route of adenosine that allows for maximal vasodilation. ${ }^{12,19-24}$ These studies have been inconclusive; some document equivalence of small doses of intracoronary adenosine and high-dose intravenous adenosine, and others document the need for extremely high doses of intracoronary adenosine or even combinations of intravenous and intracoronary adenosine to achieve a purported maximal hyperemic state. Hence, the assumption of absolute stable and minimal coronary resistance is most likely unattainable in the clinical setting, where prevailing conditions determine the magnitude to which adenosine in a given 


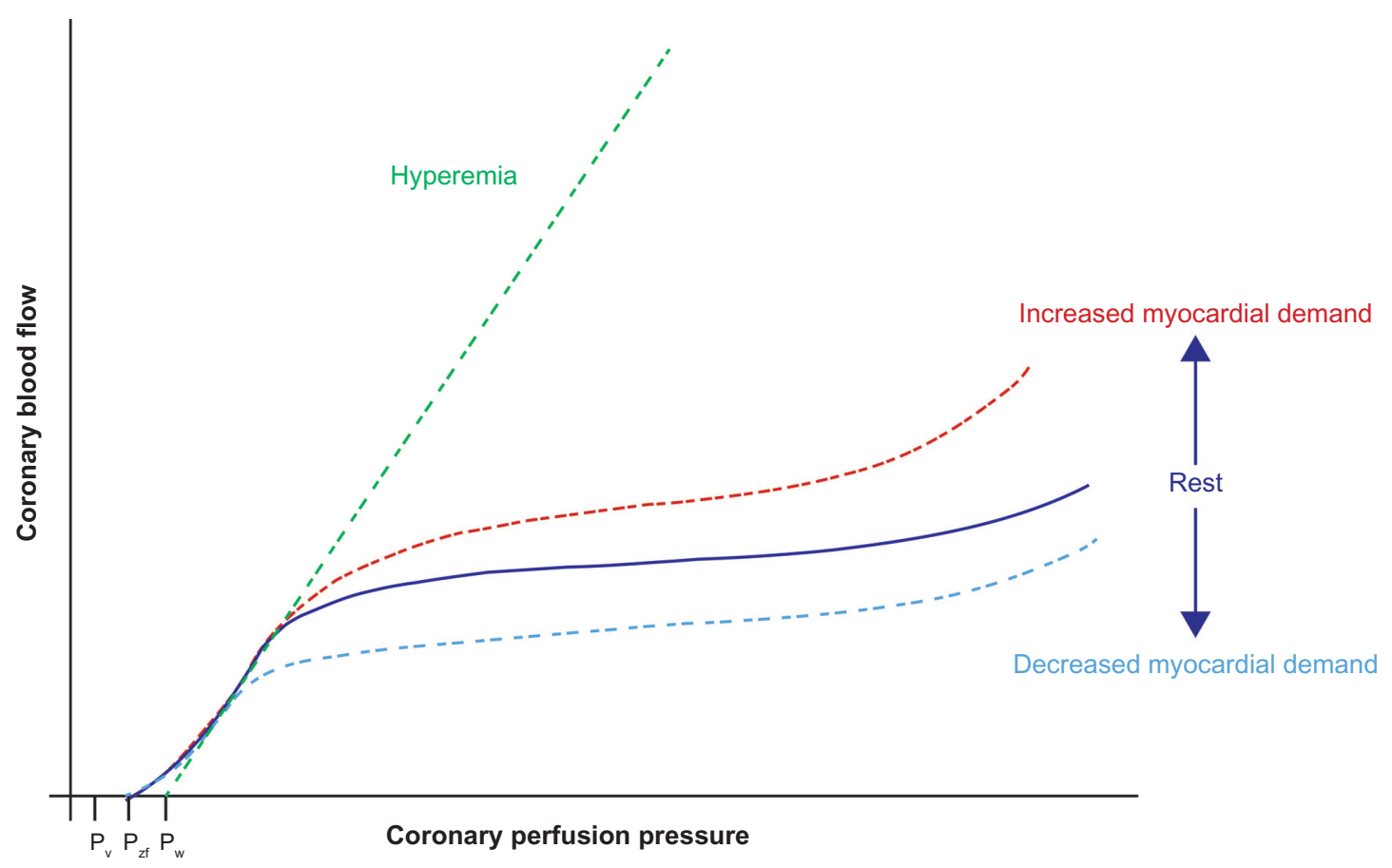

Figure I The coronary pressure-flow relationship.

Notes: Coronary blood flow at rest (solid line) is controlled by autoregulation and metabolic adaptation in order to maintain blood flow at a level that meets myocardial demand. During coronary hyperemia, autoregulatory control is exhausted and blood flow depends on perfusion pressure. The coronary pressure-flow relationship is concave at low perfusion pressures. The zero-flow intercept on the pressure axis $\left(P_{z f}\right)$ slightly exceeds venous pressure $\left(P_{v}\right)$. Straight extrapolation of the hyperemic pressure-flow relationship results in an incremental linear relationship that intercepts the pressure axis at the coronary wedge pressure $\left(\mathrm{P}_{\mathrm{w}}\right)$. Small vessel disease or abnormal left ventricular function decreases the slope of the hyperemic pressure-flow relationship, whereas elevated left ventricular end-diastolic pressure or left ventricular hypertrophy cause a parallel shift of the hyperemic pressure-flow relationship to the right.

dose is able to reduce and stabilize coronary resistance, which induces an important, but largely unappreciated, variability in FFR assessment in clinical practice.

Moreover, even if maximal hyperemia could be achieved, it is important to realize that, at this state, the relationship between coronary pressure and flow is not proportionallinear, but incremental-linear. The slope of this relationship is subject to a variety of external factors that thereby eventually determine the exact pressure-flow relation during hyperemia in the individual patient (Figure 1), which is not accounted for in the clinical application of FFR. ${ }^{25-27}$ Hence, microvascular resistance, although assumed to be stable and minimal for the pressure-derived FFR to be an accurate surrogate of blood flow impairment, is variable even at maximal vasodilation.

Furthermore, the assumption of stable and minimal microvascular resistance implies that this condition should be equally present in the absence and presence of a stenosis. Since FFR is measured in a stenotic vessel only, it is therefore assumed that removal of the stenosis would not affect the distal microvascular resistance. However, arteries are well known to be pressure-distensible, ${ }^{28}$ and it is well documented that vessel resistance to flow increases with decreasing vessel diameter. ${ }^{29}$ Accordingly, analogous to the pressure-distensibility of epicardial coronary arteries, it was documented that relief of epicardial stenosis leads to a reduction in minimal microvascular resistance, ${ }^{30-32}$ likely due to the distending effect of the increase in coronary perfusion pressure and increase in arterial inflow to the distal microvasculature. ${ }^{14,29,33}$

Obviously, these ambiguities in the physiological fundament of FFR have their reflection in the accuracy of FFR as an estimate of blood flow impairment. Even though the experimental validation of FFR documented a strong relationship between FFR and its flow-based equivalent, ${ }^{1}$ it needs to be borne in mind that much of the aforementioned variability in minimal microcirculatory resistance is influenced to a large extent by the presence of microcirculatory abnormalities. Clearly, an animal model consisting of young animals with no atherosclerosis or microvascular dysfunction will behave fundamentally differently from a routine clinical population of patients averaging over 50 years of age with both focal and diffuse atherosclerotic narrowing, as well as microvascular disease and dysfunction. Importantly, the presence of microvascular abnormalities that are associated with an increase in minimal microvascular resistance, as are present in a routine clinical population referred for 
intracoronary physiological testing, is associated with a progressive underestimation of both the true physiological severity and the ischemic potential of a coronary stenosis by FFR. ${ }^{34}$ The use of an empirically defined revascularization threshold for FFR only allows a partial correction for the aforementioned variability of the pressure-flow relation in the individual patient, which means that the optimal FFR cutoff value for revascularization decision-making is different in each individual patient and is determined by the prevailing conditions that determine the actual pressure-flow relation in the vessel under investigation. ${ }^{12}$ These considerations imply that, despite the well-documented benefit of FFR-guided revascularization over angiography guidance, FFR is not a gold-standard reference test and should be interpreted with caution in the individual patient.

\section{FFR and coronary backpressure: practical simplification versus the importance of backpressure}

In contemporary clinical practice, the ratio of mean distal coronary pressure to mean aortic pressure during maximal vasodilation is referred to as FFR and is applied so as to reflect the ratio of maximal blood flow in the target myocardium to the hypothetical maximal blood flow in the same territory in the absence of a stenosis (myocardial FFR [FFRmyo]). However, in its experimental validation, calculation of FFRmyo included correction for the central venous pressure. The latter was omitted in the clinical evaluation of FFR with the assumption that central venous pressure is usually negligible in comparison with aortic and distal coronary pressure, and this omission was governed by a practical simplification of
FFR by overcoming the requisite for a central venous catheter. Elusively increased venous pressure may, however, lead to an underestimation of true FFRmyo by FFR, and thereby alter treatment strategy for a given stenosis when strict FFR cutoff values are used..$^{35}$ Hence, although the routine application of FFR without venous pressure correction was documented to enhance clinical outcomes in the FAME and FAME 2 trials, this nuance should be borne in mind when interpreting FFR values in the individual patient. A similar dependence of FFR on left ventricular end-diastolic pressure (LVEDP) has been noted in recent literature, where elusively increased LVEDP was associated with an inadvertent increase in FFR. ${ }^{36}$ Hence, increases in coronary backpressure lead to an underestimation of true FFRmyo by its current clinical application, which adds variability to FFR and should be considered when interpreting FFR values in the individual patient.

\section{FFR-defined blood flow impairment: gold standard or imperfect surrogate?}

On many occasions, FFR is noted to reflect the fraction of flow impairment in the stenosed vessel, and its absolute value is directly extrapolated to the increase in flow expected after (perfect) coronary revascularization. For example, an FFR of 0.80 purportedly reflects that alleviation of the epicardial stenosis would allow a $20 \%$ increase in hyperemic coronary flow. However, it is important to consider that FFR is not the same as direct measures of coronary flow and flow reserve from which it was initially derived. For this purpose, it is essential to discuss the behavior of coronary hemodynamics in the absence and presence of a coronary stenosis (Figures 2 and 3). ${ }^{11,12}$ The hemodynamic

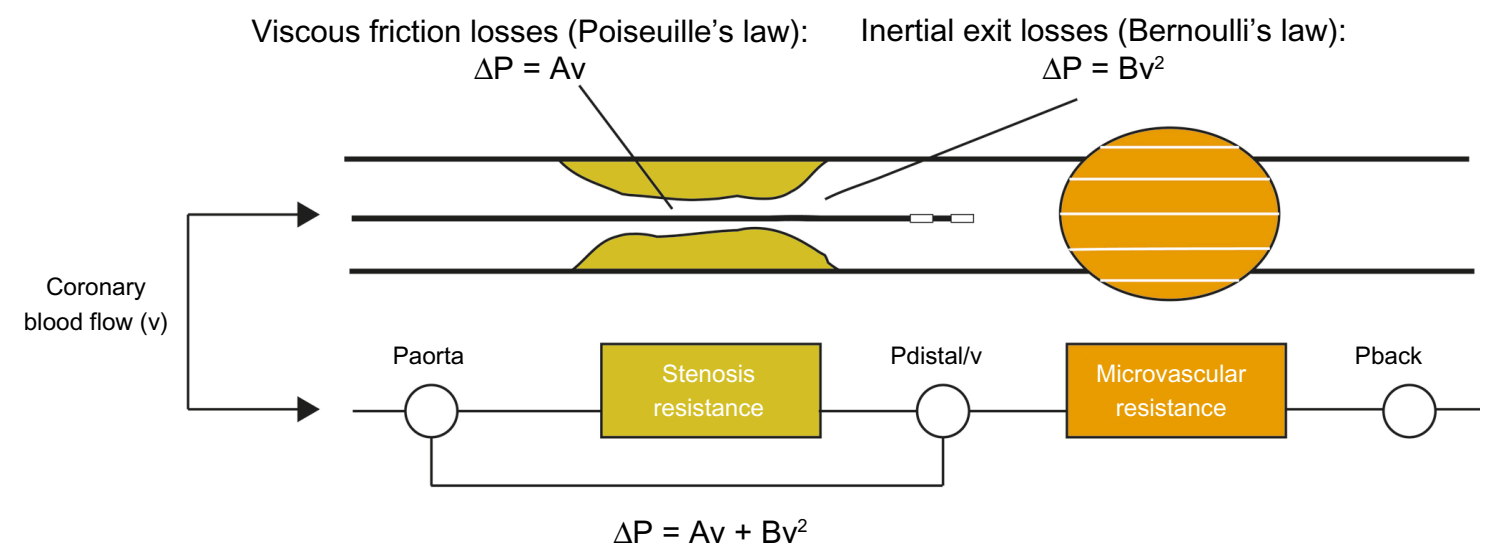

Figure 2 Resistance model of the coronary circulation.

Notes: The pressure gradient across a stenosis is determined by the sum of the stenosis' friction and exit losses. Friction losses are linearly related to the flow through the stenosis, whereas exit losses increase with the square of flow, resulting in a unique curvilinear relation between pressure gradient and flow velocity for a given stenosis geometry. Measurement of intracoronary physiology includes proximal perfusion pressure (Paorta), coronary pressure and flow velocity distal of the stenosis (Pdistal and $\mathrm{v}$, respectively), and the venous back pressure (Pback) that is usually assumed to be minimal. The relationship between blood flow through the stenosis and the resulting pressure drop is described by the quadratic equation $\Delta P=A v+B v^{2}$, where $\Delta P$ is the pressure drop across the stenosis, $v$ is flow velocity, and the coefficients $A$ and $B$ are a function of stenosis geometry and rheological properties of blood. 


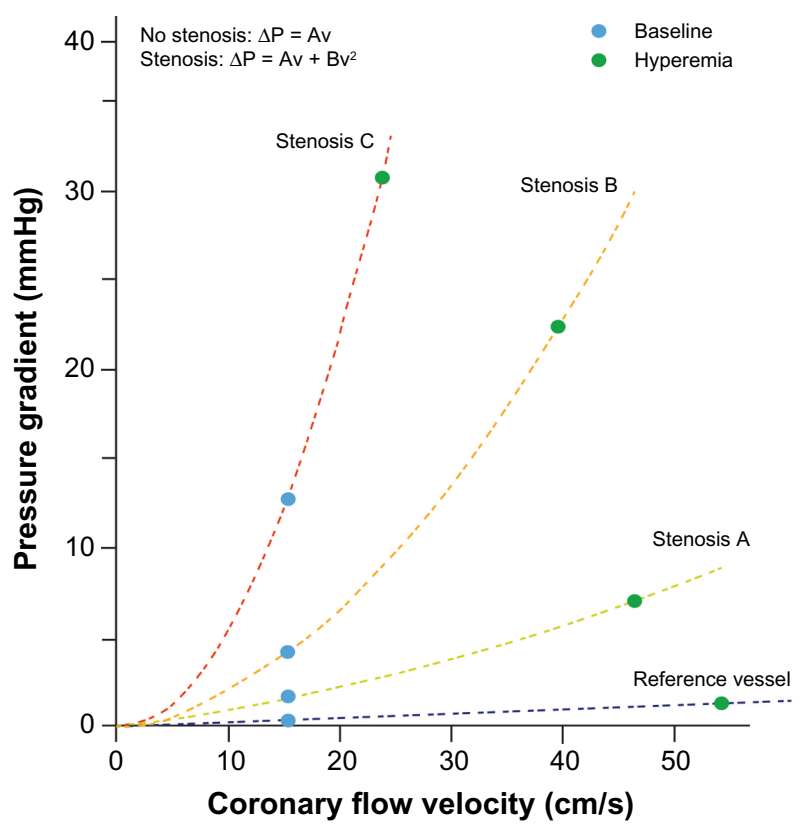

Figure 3 Pressure gradient-flow velocity relationship.

Notes: Pressure loss over an epicardial vessel without a stenosis is negligible, and therefore increases linearly with flow across the stenosis (reference vessel). With increasing stenosis severity (increasing stenosis severity from stenosis $A$ to stenosis $\mathrm{C})$, the pressure gradient-flow velocity $(\Delta \mathrm{P}-\mathrm{v})$ curve becomes steeper, reflecting incremental loss of perfusion pressure over the stenosis with increasing flow velocity with increasing stenosis severity. This $\Delta P$-v curve, the fingerprint of a given stenosis, is described by the quadratic equation $\Delta P=A v+B v^{2}$, where $\Delta P$ is the pressure drop across the stenosis, $v$ is flow velocity, and the coefficients $A$ and $B$ are a function of stenosis geometry and rheological properties of blood.

relevance of coronary stenoses is most accurately described by the relationship between the pressure drop across the stenosis and the flow that goes through it, which serves as the fingerprint of a given stenosis. The pressure losses across the stenosis occur from viscous friction across the entrance and throat of the stenosis - which increase linearly with flow through the stenosis - and flow separation losses at the stenosis exit - which increase with the square of flow. Hence, the stenosis pressure quadratically increases with an increase in coronary flow. ${ }^{37}$ In the absence of a stenosis, no quadratic flow separation losses are present, and the pressure loss along the coronary artery is linearly related to the flow that goes through it (Figure 3). This physiological background is important, since it implies that the pressure drop across a coronary stenosis, as measured for FFR, increases with an increase in coronary flow through the stenosis. In other words, with an increase in coronary flow, FFR decreases. This also explains why direct measures of coronary flow, like CFR, can disagree with the pressure-derived FFR. ${ }^{38-41}$ These disagreements have previously been attributed to technical and theoretical ambiguities associated with CFR, but it is now commonly recognized that such discordance is the result of pertinent coronary pathophysiology. Retrospectively, this is not surprising, since the accuracy of both FFR and CFR to document hemodynamically significant coronary stenoses has always been notably similar. ${ }^{42}$ Nonetheless, disagreement between FFR and CFR occurs in $30 \%-40 \%$ of coronary stenoses of intermediate angiographic severity, and thus applies to a substantial number of patients encountered in daily clinical practice. ${ }^{38,41,43}$

When both FFR and CFR are normal, the vasodilatory capacity of the coronary circulation is preserved and no hemodynamically significant stenosis occurs along the vessel (Figure 4). ${ }^{38,41}$ When both FFR and CFR are abnormal, a vastly hemodynamically significant stenosis is present that has completely exhausted the vasodilatory capacity of the coronary circulation. ${ }^{38,41}$ When FFR is abnormal and CFR is above the normal thresholds, the evaluated stenosis is non-flow-limiting: the vasodilatory capacity in the coronary circulation is preserved, and the associated high coronary flows at coronary vasodilation induce an abnormal FFR value by increasing the pressure drop across the stenosis as

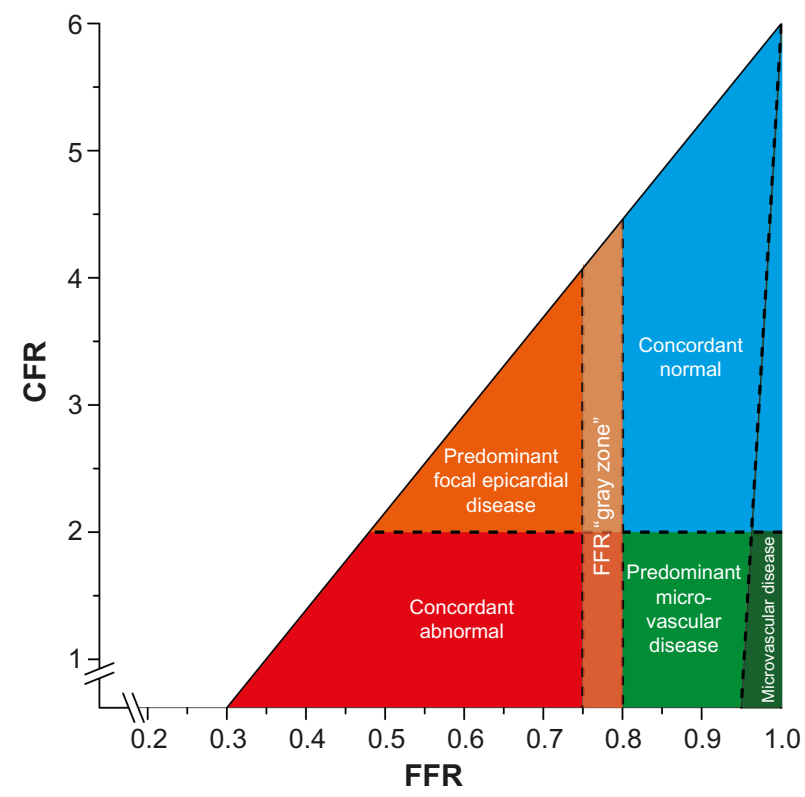

Figure 4 Conceptual plot of the fractional flow reserve (FFR)-coronary flow reserve (CFR) relationship.

Notes: Four main quadrants can be identified by applying the clinically applicable cutoff values for FFR and CFR, indicated by the dotted lines. Patients in the upperright blue area are characterized by concordantly normal FFR and CFR, and patients in the lower-left red area are characterized by concordantly abnormal FFR and CFR. Patients in the upper-left orange area and lower-right light-green area are characterized by discordant results between FFR and CFR, where the combination of an abnormal FFR and a normal CFR indicates predominant focal epicardial, but non-flow-limiting, coronary artery disease, and the combination of a normal FFR and an abnormal CFR indicates predominant microvascular involvement in coronary artery disease. The small dark-green region in the lower right is characterized by an FFR near I and an abnormal CFR, indicating sole involvement of the coronary microvasculature. The FFR "gray zone" indicates the equivocal 0.75-0.80 FFR range. Copyright $@ 2015$ by American Heart Association Inc. Adapted with permission from van de Hoef et al. van de Hoef TP, van Lavieren MA, Damman P, et al. Physiological basis and long-term clinical outcome of discordance between fractional flow reserve and coronary flow velocity reserve in coronary stenoses of intermediate severity. Circ Cardiovasc Interv. 2014; 7(3):301-311. ${ }^{38}$ 
explained earlier. ${ }^{38,41}$ Despite having abnormal FFR values, these vessels are likely not optimally managed by coronary revascularization. ${ }^{38}$ When FFR is normal and CFR is impaired, three explanations may (co)exist. First, this may be a representation of sole microvascular disease, which is likely when CFR is reduced and FFR approaches normal values. Second, this may be a reflection of a mild epicardial stenosis superimposed on a background of diffuse or microcirculatory disease. Finally, it may also be the case that a hemodynamically important stenosis is present, accompanied by vast microcirculatory disease. ${ }^{38,41}$ Since microvascular disease may limit the maximal hyperemic flow through a stenosis, the true hemodynamic importance of the epicardial stenosis may be underestimated by FFR. ${ }^{34}$ Analogous to earlier findings on the dominant prognostic importance of CFR over measures of FFR derived from noninvasive studies, ${ }^{44-46}$ observational data have now suggested that CFR dictates clinical outcomes after deferral of revascularization. ${ }^{38}$ These findings have led to the initiation of a large multicenter study on the prognostic relevance of FFR/CFR discordance - DEFINE FLOW (NCT02328820) - in which revascularization will be deferred in all stenoses associated with normal FFR and CFR values, as well as in those stenoses with discordant FFR and CFR values. The primary outcome-2-year major adverse cardiac events (MACE) rate - is eagerly awaited.

\section{FFR to guide revascularization}

After the introduction of FFR based on validation work in an animal model, ${ }^{1}$ several clinical studies were performed to assess the relationship between FFR and the occurrence of inducible myocardial ischemia in order to identify its potential for revascularization decision-making. ${ }^{10,47}$ After studies defining the relationship of FFR with electrical manifestations of myocardial ischemia on exercise stress testing ${ }^{48}$ and with positron emission tomography-defined flow abnormalities, ${ }^{8}$ Pijls et al defined the optimal FFR cutoff value for inducible myocardial ischemia by means of a study using a multi-test reference standard composed of exercise electrocardiography, dobutamine stress echocardiography, and myocardial perfusion scintigraphy. ${ }^{49}$ Despite the limited sample size of only 45 patients, and concerns about the study methodology, the optimal 0.75 FFR cutoff value documented in this study is now widely accepted as the optimal FFR cutoff value of 0.75 for inducible myocardial ischemia. ${ }^{50}$ The first clinical decisionmaking studies, among which is the pivotal Deferral Versus Performance of PTCA in Patients Without Documented Ischemia (DEFER) study, used this 0.75 FFR cutoff value of 0.75 to guide revascularization decisions. Most importantly, the
DEFER study documented that deferral of revascularization of stenoses with an FFR $\geq 0.75$ was safe as it was associated with a reduction in the occurrence of MACE compared with revascularization of these stenoses. ${ }^{51,52}$ Regardless of the work preceding adoption of the 0.75 FFR cutoff, and the clinical data supporting the 0.75 FFR cutoff value, further randomized evaluation of FFR-guided percutaneous coronary intervention (PCI) against angiography-guided PCI used an elevated 0.80 FFR cutoff value. ${ }^{2,5,6}$ The randomized FAME study compared FFR-guided PCI at the $0.80 \mathrm{FFR}$ cutoff value with angiography-guided PCI in terms of the occurrence of MACE. ${ }^{2}$ The use of FFR to guide clinical decision-making was documented to improve clinical outcomes compared with an angiography-guided approach while limiting the number of stents and total stent length used. ${ }^{2}$ This laid the fundament of a functional approach to coronary revascularization by illustrating that relief of functionally nonsignificant coronary disease, as assessed by coronary pressure measurements, does not improve clinical outcome and leads to overutilization of resources. Moreover, an ancillary study documented the superior cost-effectiveness of an FFR-guided strategy over an angiography-guided approach, ${ }^{53}$ further documenting the requisite of a functional rather than visual approach to epicardial coronary revascularization. Despite these favorable results, the use of FFR to guide decision-making remained limited, ${ }^{54}$ likely in part due to the practical cumbersomeness of FFR measurement in all eligible PCI cases, but also in part due to the fact that a benefit of PCI over medical therapy had never been established in the setting of stable ischemic heart disease. ${ }^{55}$

The FAME 2 study evaluated the clinical benefit of mechanical revascularization in stenoses deemed functionally significant at the FFR 0.80 cutoff value. ${ }^{56}$ Those stenoses with an FFR $\leq 0.80$ were randomized to optimal medical therapy plus PCI or optimal medical therapy alone, while those stenoses with an FFR $>0.80$ were not randomized and were considered a reference group, treated by means of optimal medical therapy. By these means, FAME 2 documented that mechanical relief of stenoses deemed significant by an FFR at the 0.80 cutoff value is associated with a reduction in the occurrence of MACE. ${ }^{5,6}$ At 2 years, the investigators also documented a slight benefit of PCI in FFR-positive stenoses in terms of hard clinical endpoints, cardiac death, and myocardial infarction. ${ }^{6}$ However, it should be noted that the FAME 2 trial was halted early due to the dominant benefit of PCI in FFR-positive stenosis on the occurrence of the composite MACE endpoint, limiting the trial's statistical power and giving rise to a potential overestimation of 
effect size. ${ }^{5}$ Moreover, detailed evaluation of the FAME 2 data reveals important flipsides. First, although a significant difference was documented between the PCI plus optimal medical therapy versus optimal medical therapy only arms in terms of MACE, it is important to realize that the majority of FFR-positive stenoses that were treated by optimal medical therapy alone did not require revascularization or suffered from a hard clinical endpoint at up to 2 years of follow-up. Moreover, in the reference group - those with stenoses with FFR greater than 0.80 who were treated by optimal medical therapy alone - still $>10 \%$ of vessels suffered MACE in the first 2 years of follow-up. These data illustrate that the majority of FFR-positive vessels are not at risk for adverse events, and a substantial proportion of FFR-negative vessels are adversely at high risk for MACE. These data effectively contradict the extremely high accuracy of FFR for inducible myocardial ischemia documented in the original multi-test ischemia study ${ }^{49}$ and raise concerns regarding contemporary revascularization guidelines in which all FFR-positive stenoses are pondered alike and deemed eligible for coronary revascularization. ${ }^{57,58}$

\section{Physiological ambiguities of FFR and FAME 2 results: a synopsis}

Effectively, the FAME 2 study documented that a majority of stenoses deemed hemodynamically significant by FFR revascularization could be deferred based on the clinical outcomes, even though all of these stenoses are considered eligible for revascularization in contemporary coronary revascularization guidelines. ${ }^{5,6}$ These findings are notable when interpreted in the light of the extremely high sensitivity and specificity for myocardial ischemia documented in its validation studies, ${ }^{49}$ but not surprising when considering the sensitivity and specificity of FFR for myocardial ischemia in a more variable clinical setting ${ }^{47}$ or prevalence of FFR discordance with CFR. ${ }^{38,41}$ While both noninvasive studies and observational invasive data have shown dominance of CFR over FFR for clinical outcomes after deferral of revascularization, ${ }^{38,44-46}$ preclinical data also support this hypothesis by documenting that myocardial function thrives on coronary blood flow and not on perfusion pressure. ${ }^{59}$ As such, the stenoses deemed hemodynamically important by FFR in which revascularization is deferred, but which do not evolve to develop adverse events, may be hypothesized to form the group of stenoses in which the low FFR is discordant with a normal CFR. This hypothesis is supported both by the validation data on FFR as well as observational data on the relationship of FFR with clinical outcomes. First, it was documented that impaired coronary flow characteristics in the vessel of interest are largely associated with FFR values below $0.65 .{ }^{60,61}$ A large patient-level pooled analysis further noted a 0.66 FFR cutoff value to be the FFR cut-point below which coronary revascularization is likely to improve patient outcomes over optimal medical therapy. ${ }^{62}$ Moreover, FAME 2 documented that the coronary revascularization was dominantly associated with improved clinical benefit in vessels with an FFR $<0.65 .^{5}$ These cutoff values are notably similar to the FFR cutoff value proposed in the validation study of FFR against signs of ischemia on exercise stress electrocardiography - a test known for its high specificity for myocardial ischemia. ${ }^{48}$ This study also documented a 0.66 FFR cutoff value for discrimination of inducible myocardial ischemia, while all subsequent studies have since led to a stepwise increase in the clinically applied FFR cutoff. ${ }^{49,56}$ It is therefore likely that stenoses with an FFR $<0.65$ indeed require revascularization to improve patient outcomes because they are associated with impairment of coronary flow characteristics of the vasculature of interest that are ultimately responsible for the occurrence of signs and symptoms of myocardial ischemia. ${ }^{60}$ Conversely, stenoses with an FFR $>0.65$ are likely optimally managed by medical therapy if they are not objectively associated with impairment of direct measures of coronary flow.

The implications of the clinical outcomes of the reference group in the FAME 2 study are frequently overlooked. ${ }^{6}$ Within those patients in whom FFR values were normal, the event rate at 2-year follow-up exceeded $10 \%$. These data imply that a normal FFR does not reflect a normal vasculature, and that patients with normal FFR values may still be at increased risk for adverse events. The discordance of a normal FFR and abnormal CFR may explain this finding. Although less common, this type of discordance is associated with a high rate of adverse cardiac events in the first years of follow-up. ${ }^{38,44}$ These patients are not identified in contemporary practice, in which all normal FFR values are pondered alike, even though their identification seems important in terms of clinical outcomes.

\section{Flow-based evaluation of ischemic heart disease: a glimpse into the future?}

Considering the dominance of coronary flow impairment over impairment of FFR for clinical outcomes, as well as the interpretation of the aforementioned FAME 2 study outcomes, a flow-based primary approach in stable ischemic heart disease might improve patient selection for revascularization. In such an approach, invasive or noninvasive flow evaluation should serve as a gatekeeper by identification of abnormal flow characteristics in the individual patient. If flow impairment 
is present, patients should be further evaluated to identify the presence of focal epicardial disease and its eligibility for revascularization, as well as the presence of microcirculatory abnormalities. Patients in whom no flow abnormalities are documented are likely optimally managed by medical therapy, and no further (invasive) evaluation should be necessary. With such an approach, procedural risks are directed toward those patients in whom the likeliness of clinical benefit is highest, and costly procedures are avoided in patients in whom more detailed insight into the coronary circulation does not bear relevance for treatment strategies. The possibilities for such an approach are multiple, and may well improve diagnosis and risk stratification of stable ischemic heart disease patients.

One such approach may be found in the concept of coronary flow capacity, which uses the relationship between CFR (the ratio of hyperemic to basal flow [velocity]) and hyperemic flow to document the ischemic potential of the vasculature under investigation. The concept of coronary flow capacity was first validated using positron emission tomography to document flow, where it was documented to be closely related to objective signs and symptoms of myocardial ischemia. ${ }^{60,61}$ Second, the concept was validated using invasive Doppler flow measurements, where it was documented to provide incremental value for risk stratification of stable coronary artery disease patients with intermediate coronary stenosis over both CFR as well as FFR. As such, although still in the early stages of its development, coronary flow capacity may provide a valuable tool for identifying the ischemic burden of the vasculature under investigation and improve risk stratification of stable ischemic heart disease patients before more stenosis-specific tools are employed to identify revascularization targets or microvascular dysfunction as its primary cause. ${ }^{63}$

\section{Conclusion}

FFR remains the most well-studied physiological parameter to guide coronary revascularization decision-making in contemporary practice, and it is the sole parameter documented to date to outperform visual guidance of coronary revascularization. Notwithstanding, the clinical performance of FFR against coronary angiography is no proof of its physiological fundament, and its associated ambiguities need to be considered in clinical practice. As such, the FAME 2 study provides essential insight into the ambiguities associated with FFR-guided coronary revascularization, documenting that FFR is far from able to optimally identify patients at high risk for adverse cardiac events. In this regard, its physiological basis provides an explanation that is dictated by the imperfect relationship of FFR with direct measures of coronary flow and flow reserve from which it was derived, and which critically determine the occurrence of signs and symptoms of myocardial ischemia, and seem to dominate clinical outcomes after deferral of coronary revascularization. All evidence gathered, it seems that a flow-based primary evaluation of stable ischemic heart disease patients may improve patient selection for revascularization and help optimize outcomes of physiology-guided decision-making.

\section{Disclosure}

Dr van de Hoef has served as a speaker at educational events organized by Volcano Corporation, St Jude Medical, and Boston Scientific, manufacturers of sensor-equipped guide wires. Drs Meuwissen and Piek have served as speakers at educational events organized by Volcano Corporation. The authors report no other conflicts of interest in this work.

\section{References}

1. Pijls NH, van Son JA, Kirkeeide RL, De Bruyne B, Gould KL. Experimental basis of determining maximum coronary, myocardial, and collateral blood flow by pressure measurements for assessing functional stenosis severity before and after percutaneous transluminal coronary angioplasty. Circulation. 1993;87(4):1354-1367.

2. Tonino PA, De Bruyne B, Pijls NH, et al; FAME Study Investigators. Fractional flow reserve versus angiography for guiding percutaneous coronary intervention. N Engl J Med. 2009;360(3):213-224.

3. Pijls NH, Fearon WF, Tonino PA, et al; FAME Study Investigators. Fractional flow reserve versus angiography for guiding percutaneous coronary intervention in patients with multivessel coronary artery disease: 2-year follow-up of the FAME (Fractional Flow Reserve Versus Angiography for Multivessel Evaluation) study. J Am Coll Cardiol. 2010;56(3):177-184.

4. Bech GJ, De Bruyne B, Pijls NH, et al. Fractional flow reserve to determine the appropriateness of angioplasty in moderate coronary stenosis: a randomized trial. Circulation. 2001;103(24):2928-2934.

5. De Bruyne B, Pijls NH, Kalesan B, et al; FAME 2 Trial Investigators. Fractional flow reserve-guided PCI versus medical therapy in stable coronary disease. N Engl J Med. 2012;367(11):991-1001.

6. De Bruyne B, Fearon WF, Pijls NH, et al; FAME 2 Trial Investigators. Fractional flow reserve-guided PCI for stable coronary artery disease. N Engl J Med. 2014;371(13):1208-1217.

7. Young DF, Cholvin NR, Kirkeeide RL, Roth AC. Hemodynamics of arterial stenoses at elevated flow rates. Circ Res. 1977;41(1):99-107.

8. De Bruyne B, Baudhuin T, Melin JA, et al. Coronary flow reserve calculated from pressure measurements in humans. Validation with positron emission tomography. Circulation. 1994;89(3):1013-1022.

9. Gould KL, Johnson NP, Bateman TM, et al. Anatomic versus physiologic assessment of coronary artery disease. Role of coronary flow reserve, fractional flow reserve, and positron emission tomography imaging in revascularization decision-making. J Am Coll Cardiol. 2013; 62(18):1639-1653.

10. van de Hoef TP, van Lavieren MA, Henriques JP, Piek JJ, Claessen BE. Fractional flow reserve-guided percutaneous coronary intervention: does coronary pressure never lie? Curr Treat Options Cardiovasc Med. 2014;16(4):294.

11. van de Hoef TP, Nolte F, Rolandi MC, et al. Coronary pressure-flow relations as basis for the understanding of coronary physiology. $\mathrm{J} \mathrm{Mol}$ Cell Cardiol. 2012;52(4):786-793.

12. van de HoefTP, Meuwissen M, Escaned J, et al. Fractional flow reserve as a surrogate for inducible myocardial ischaemia. Nat Rev Cardiol. 2013;10(8):439-452. 
13. Pijls NH, Kern MJ, Yock PG, De Bruyne B. Practice and potential pitfalls of coronary pressure measurement. Catheter Cardiovasc Interv. 2000;49(1):1-16.

14. Spaan JA, Piek JJ, Hoffman JI, Siebes M. Physiological basis of clinically used coronary hemodynamic indices. Circulation. 2006; 113(3):446-455.

15. Pijls NH, Sels JW. Functional measurement of coronary stenosis. J Am Coll Cardiol. 2012;59(12):1045-1057.

16. Heusch G. Adenosine and maximum coronary vasodilation in humans: myth and misconceptions in the assessment of coronary reserve. Basic Res Cardiol. 2010;105(1):1-5.

17. Barbato E, Bartunek J, Aarnoudse W, et al. Alpha-adrenergic receptor blockade and hyperaemic response in patients with intermediate coronary stenoses. Eur Heart J. 2004;25(22):2034-2039.

18. Aarnoudse W, Geven M, Barbato E, Botman KJ, De Bruyne B, Pijls NH. Effect of phentolamine on the hyperemic response to adenosine in patients with microvascular disease. Am J Cardiol. 2005;96(12): $1627-1630$.

19. De Luca G, Venegoni L, Iorio S, Giuliani L, Marino P. Effects of increasing doses of intracoronary adenosine on the assessment of fractional flow reserve. JACC Cardiovasc Interv. 2011;4(10):1079-1084.

20. De Bruyne B, Pijls NH, Barbato E, et al. Intracoronary and intravenous adenosine 5 -triphosphate, adenosine, papaverine, and contrast medium to assess fractional flow reserve in humans. Circulation. 2003;107: 1877-1883.

21. Jeremias A, Whitbourn RJ, Filardo SD, et al. Adequacy of intracoronary versus intravenous adenosine-induced maximal coronary hyperemia for fractional flow reserve measurements. Am Heart J. 2000; 140(4):651-657.

22. Kern MJ, Deligonul U, Tatineni S, Serota H, Aguirre F, Hilton TC. Intravenous adenosine: continuous infusion and low dose bolus administration for determination of coronary vasodilator reserve in patients with and without coronary artery disease. J Am Coll Cardiol. 1991;18(3):718-729.

23. Türkoğlu C, Oztürk M, Aliyev F, Firatli I, Incesoy N. Electrophysiologic characteristics of wide QRS complexes during pharmacologic termination of sustained supraventricular tachycardias with verapamil and adenosine: observations from electrophysiologic study. Ann Noninvasive Electrocardiol. 2009;14(4):375-380.

24. Schlundt C, Bietau C, Klinghammer L, et al. Comparison of intracoronary versus intravenous administration of adenosine for measurement of coronary fractional flow reserve. Circ Cardiovasc Interv. 2015;8(5).

25. Duncker DJ, Bache RJ. Effect of chronotropic and inotropic stimulation on the coronary pressure-flow relation in left ventricular hypertrophy. Basic Res Cardiol. 1997;92(4):271-286.

26. Jeremy RW, Hughes CF, Fletcher PJ. Effects of left ventricular diastolic pressure on the pressure-flow relation of the coronary circulation during physiological vasodilatation. Cardiovasc Res. 1986;20(12): 922-930.

27. Duncker DJ, Zhang J, Pavek TJ, Crampton MJ, Bache RJ. Effect of exercise on coronary pressure-flow relationship in hypertrophied left ventricle. Am J Physiol. 1995;269(1 Pt 2):H271-H281.

28. Muller O, Pyxaras SA, Trana C, et al. Pressure-diameter relationship in human coronary arteries. Circ Cardiovasc Interv. 2012;5(6): 791-796.

29. Kanatsuka H, Ashikawa K, Komaru T, Suzuki T, Takishima T. Diameter change and pressure-red blood cell velocity relations in coronary microvessels during long diastoles in the canine left ventricle. Circ Res. 1990;66(2):503-510.

30. Verhoeff BJ, Siebes M, Meuwissen M, et al. Influence of percutaneous coronary intervention on coronary microvascular resistance index. Circulation. 2005;111(1):76-82.

31. Chamuleau SA, Siebes M, Meuwissen M, Koch KT, Spaan JA, Piek JJ. Association between coronary lesion severity and distal microvascular resistance in patients with coronary artery disease. Am J Physiol Heart Circ Physiol. 2003;285(5):H2194-H2200.
32. Indermühle A, Vogel R, Meier P, Zbinden R, Seiler C. Myocardial blood volume and coronary resistance during and after coronary angioplasty. Am J Physiol Heart Circ Physiol. 2011;300(3):H1119-H1124.

33. Hanley FL, Messina LM, Grattan MT, Hoffman IE. The effect of coronary inflow pressure on coronary vascular resistance in the isolated dog heart. Circ Res. 1984;54(6):760-772.

34. van de Hoef TP, Nolte F, EchavarrÍa-Pinto M, et al. Impact of hyperaemic microvascular resistance on fractional flow reserve measurements in patients with stable coronary artery disease: insights from combined stenosis and microvascular resistance assessment. Heart. 2014;100(12):951-959.

35. Perera D, Biggart S, Postema P, et al. Right atrial pressure: can it be ignored when calculating fractional flow reserve and collateral flow index? J Am Coll Cardiol. 2004;44(10):2089-2091.

36. Leonardi RA, Townsend JC, Patel CA, et al. Left ventricular enddiastolic pressure affects measurement of fractional flow reserve. Cardiovasc Revasc Med. 2013;14(4):218-222.

37. Gould KL. Pressure-flow characteristics of coronary stenoses in unsedated dogs at rest and during coronary vasodilation. Circ Res. 1978; 43(2):242-253.

38. van de HoefTP, van Lavieren MA, Damman P, et al. Physiological basis and long-term clinical outcome of discordance between fractional flow reserve and coronary flow velocity reserve in coronary stenoses of intermediate severity. Circ Cardiovasc Interv. 2014; 7(3):301-311.

39. Echavarria-Pinto M, Escaned J, Macías E, et al. Disturbed coronary hemodynamics in vessels with intermediate stenoses evaluated with fractional flow reserve: a combined analysis of epicardial and microcirculatory involvement in ischemic heart disease. Circulation. 2013;128(24):2557-2566

40. Echavarría-Pinto M, van de Hoef TP, Serruys PW, Piek JJ, Escaned J. Facing the complexity of ischaemic heart disease with intracoronary pressure and flow measurements: beyond fractional flow reserve interrogation of the coronary circulation. Curr Opin Cardiol. 2014; 29(6):564-570.

41. Johnson NP, Kirkeeide RL, Gould KL. Is discordance of coronary flow reserve and fractional flow reserve due to methodology or clinically relevant coronary pathophysiology? JACC Cardiovasc Imaging. 2012; 5(2):193-202.

42. Meuwissen M, Siebes M, Chamuleau SAJ, et al. Role of fractional and coronary flow reserve in clinical decision making in intermediate coronary lesions. Interv Cardiol (Lond). 2009;1(2):237-255.

43. Meuwissen M, Chamuleau SA, Siebes M, et al. Role of variability in microvascular resistance on fractional flow reserve and coronary blood flow velocity reserve in intermediate coronary lesions. Circulation. 2001;103(2):184-187.

44. Herzog BA, Husmann L, Valenta I, et al. Long-term prognostic value of $13 \mathrm{~N}$-ammonia myocardial perfusion positron emission tomography added value of coronary flow reserve. J Am Coll Cardiol. 2009; 54(2):150-156.

45. Murthy VL, Naya M, Foster CR, et al. Improved cardiac risk assessment with noninvasive measures of coronary flow reserve. Circulation. 2011;124(20):2215-2224.

46. Murthy VL, Naya M, Foster CR, et al. Association between coronary vascular dysfunction and cardiac mortality in patients with and without diabetes mellitus. Circulation. 2012;126(15):1858-1868.

47. Christou MA, Siontis GC, Katritsis DG, Ioannidis JP. Meta-analysis of fractional flow reserve versus quantitative coronary angiography and noninvasive imaging for evaluation of myocardial ischemia. Am J Cardiol. 2007;99(4):450-456.

48. De Bruyne B, Bartunek J, Sys SU, Heyndrickx GR. Relation between myocardial fractional flow reserve calculated from coronary pressure measurements and exercise-induced myocardial ischemia. Circulation. 1995;92(1):39-46.

49. Pijls NH, De Bruyne B, Peels K, et al. Measurement of fractional flow reserve to assess the functional severity of coronary-artery stenoses. N Engl J Med. 1996;334(26):1703-1708.

50. Pijls NH, Tanaka N, Fearon WF. Functional assessment of coronary stenoses: can we live without it? Eur Heart J. 2013;34(18):1335-1344. 
51. Pijls NH, van Schaardenburgh P, Manoharan G, et al. Percutaneous coronary intervention of functionally nonsignificant stenosis: 5-year followup of the DEFER Study. J Am Coll Cardiol. 2007;49(21):2105-2111.

52. Bech GJ1, De Bruyne B, Pijls NH, et al. Fractional flow reserve to determine the appropriateness of angioplasty in moderate coronary stenosis: a randomized trial. Circulation. June 19, 2001;103(24):2928-2934.

53. Fearon WF, Bornschein B, Tonino PA, et al; Fractional Flow Reserve Versus Angiography for Multivessel Evaluation (FAME) Study Investigators. Economic evaluation of fractional flow reserve-guided percutaneous coronary intervention in patients with multivessel disease. Circulation. 2010;122(24):2545-2550.

54. Dattilo PB, Prasad A, Honeycutt E, Wang TY, Messenger JC. Contemporary patterns of fractional flow reserve and intravascular ultrasound use among patients undergoing percutaneous coronary intervention in the United States: insights from the National Cardiovascular Data Registry. J Am Coll Cardiol. 2012;60(22):2337-2339.

55. Boden WE, O'Rourke RA, Teo KK, et al. Optimal medical therapy with or without PCI for stable coronary disease. $N \mathrm{Engl} \mathrm{J} \mathrm{Med.}$ 2007;356(15):1503-1516

56. Fearon WF, Tonino PA, De Bruyne B, Siebert U, Pijls NH; FAME Study Investigators. Rationale and design of the Fractional Flow Reserve versus Angiography for Multivessel Evaluation (FAME) study. Am Heart J. 2007;154(4):632-636.

57. Windecker S, Kolh P, Alfonso F, et al. 2014 ESC/EACTS guidelines on myocardial revascularization. EuroIntervention. 2015;10(9): 1024-1094.
58. Task Force Members, Montalescot G, Sechtem U, et al. 2013 ESC guidelines on the management of stable coronary artery disease: the Task Force on the management of stable coronary artery disease of the European Society of Cardiology. Eur Heart J. 2013;34(38):2949-3003.

59. Smalling RW, Kelley K, Kirkeeide RL, Fisher DJ. Regional myocardial function is not affected by severe coronary depressurization provided coronary blood flow is maintained. J Am Coll Cardiol. 1985;5(4):948-955.

60. Johnson NP, Gould KL. Physiological basis for angina and ST-segment change PET-verified thresholds of quantitative stress myocardial perfusion and coronary flow reserve. JACC Cardiovasc Imaging. 2011; 4(9):990-998.

61. Johnson NP, Gould KL. Integrating noninvasive absolute flow, coronary flow reserve, and ischemic thresholds into a comprehensive map of physiological severity. JACC Cardiovasc Imaging. 2012;5(4): 430-440.

62. Johnson NP, Tóth GG, Lai D, et al. Prognostic value of fractional flow reserve: linking physiologic severity to clinical outcomes. J Am Coll Cardiol. 2014;64(16):1641-1654.

63. van de Hoef TP, Siebes M, Spaan JA, Piek JJ. Fundamentals in clinical coronary physiology: why coronary flow is more important than coronary pressure. Eur Heart J. Epub 2015 Jun 1.
Vascular Health and Risk Management

\section{Publish your work in this journal}

Vascular Health and Risk Management is an international, peerreviewed journal of therapeutics and risk management, focusing on concise rapid reporting of clinical studies on the processes involved in the maintenance of vascular health; the monitoring, prevention and treatment of vascular disease and its sequelae; and the involvement of

\section{Dovepress}

metabolic disorders, particularly diabetes. This journal is indexed on PubMed Central and MedLine. The manuscript management system is completely online and includes a very quick and fair peer-review system, which is all easy to use. Visit http://www.dovepress.com/ testimonials.php to read real quotes from published authors. 